Proceedings of the CSMAG'07 Conference, Košice, July 9-12, 2007

\title{
Corrosion Influence on the Magnetic Structure of Fe-Based Nanocrystalline Alloys
}

\author{
J. Sitek, K. Sedlackova and M. Pavlovic \\ Department of Nuclear Physics and Technology \\ Slovak University of Technology \\ Ilkovicova 3, 81219 Bratislava, Slovakia
}

\begin{abstract}
Nanocrystalline alloy of FINEMET and NANOPERM-type were studied by the Mössbauer spectroscopy after atmospheric corrosion up to 10 months. Corrosion process has an influence on the magnetic microstructure, which reflects at the Mössbauer parameters as a change in direction of net magnetic moment, intensity, and distribution of internal magnetic field. During corrosion process the most occurring tendency was the rotation of net magnetic moment out of the ribbon plane. This behaviour might result from the internal stress produced during corrosion treatment. The observed changes in the average hyperfine field and shape of its distribution indicated the changes of the microscopic magnetic properties induced by corrosion damage. These results are compared with radiation corrosion.
\end{abstract}

PACS numbers: $75.50 . \mathrm{Kj}, 81.40 . \mathrm{Np}$

\section{Introduction}

Iron based nanocrystalline alloys show a good combination of the low coercive force and high permeability. So far, attention has been given to the iron based nanocrystalline alloys FINEMET (FeCuNbSiB) and NANOPERM (FeZrB) concerning microstructure, crystallisation and magnetic properties [1]. However, less attention has been given to their corrosion behaviour. Corrosion resistance of amorphous and nanocrystalline alloys has been reported only partially [2, 3], but it is important for some special applications like magnetic shielding, sensors, transformer corns, etc. Radiation corrosion is relevant in applications in accelerator technology. Data exists mainly for neutrons [4], irradiation by heavy ions is in progress. In this study we report the relation between corrosion products after atmospheric corrosion and their influence on the magnetic parameters of some iron based nanocrystalline alloys.

\section{Experimental}

Three different samples were studied: FINEMET $\left(\mathrm{Fe}_{73.5} \mathrm{Cu}_{1} \mathrm{Nb}_{3} \mathrm{Si}_{13.5} \mathrm{~B}_{9}\right)$ and two types of NANOPERM ( $\mathrm{Fe}_{87.5} \mathrm{Zr}_{6.5} \mathrm{~B}_{6}$ and $\left.\mathrm{Fe}_{76} \mathrm{Mo}_{8} \mathrm{Cu}_{1} \mathrm{~B}_{15}\right)$. All samples 
were prepared in the form of ribbons from amorphous precursors by annealing at $540^{\circ} \mathrm{C}$ for one hour in vacuum. According to our experience different annealing conditions do not have an essential influence on corrosion process. The samples were exposed to real outdoor-weather conditions continuously during one year and measured repeatedly every two months. The measurements were performed at room temperature by standard transmission Mössbauer spectroscopy.

\section{Results and discussion}

The Mössbauer spectra of NANOPERM changed drastically as early as after two months. New paramagnetic phase of $\gamma-\mathrm{FeOOH}$ was created as well as pronounced changes in the bulk structural arrangement occurred. This can be seen from the shape of the Mössbauer spectra and distribution of hyperfine magnetic field of amorphous component $P(B)$, see Fig. 1.
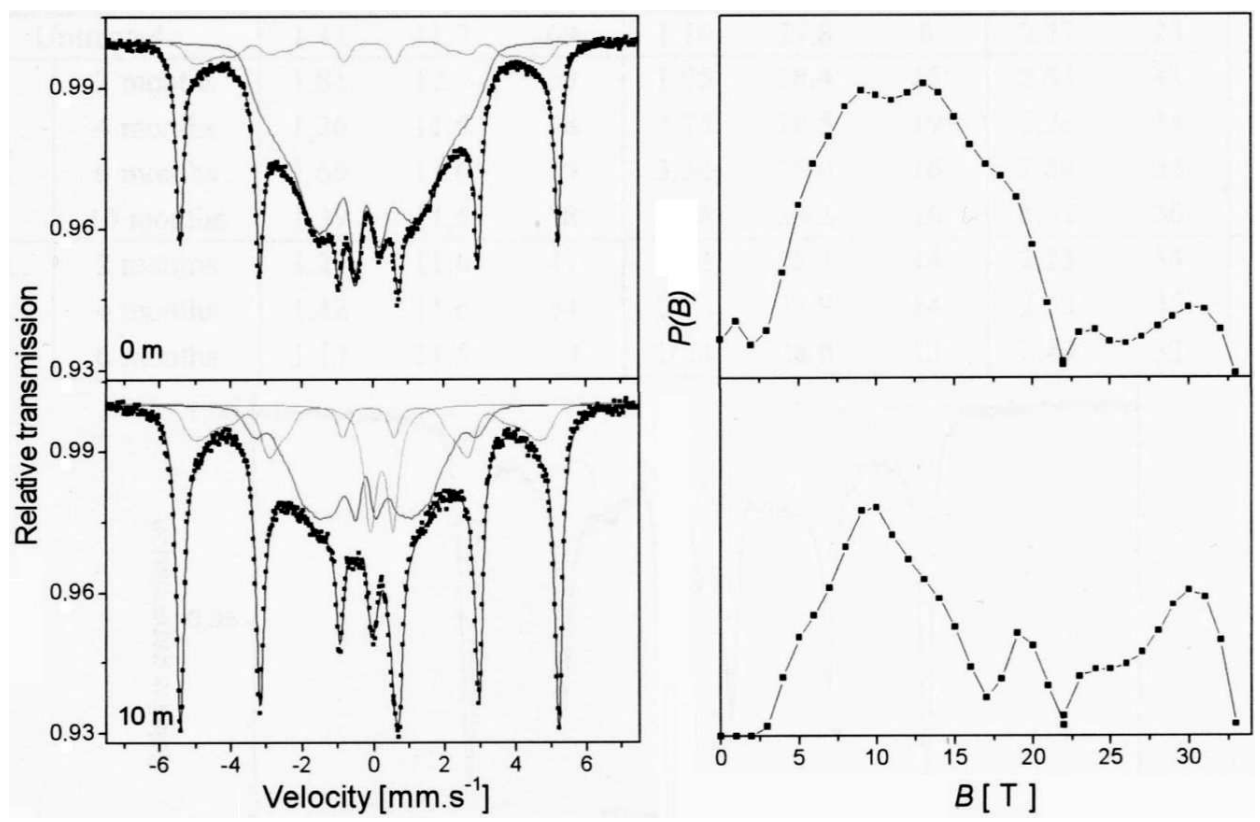

Fig. 1. Mössbauer spectra of $\mathrm{Fe}_{87.5} \mathrm{Zr}_{6.5} \mathrm{~B}_{6}: 0 \mathrm{~m}$ - without corrosion treatment; $10 \mathrm{~m}$ — after 10 months treatment; right — corresponding $P(B)$ distributions.

The re-arrangement of the constituent components indicates that atmospheric corrosion might induce crystallisation in nanocrystalline alloy leading to growth of new crystalline grains. The hyperfine field distribution confirms this tendency. The original hump of the distribution of the amorphous reminder is split into more separated peaks at lower $P(B)$ values after exposure. Correlation of these effects with macroscopic magnetic properties has been studied in [5]. 
Another Mössbauer parameter $A_{23}$ reflects the direction of net magnetic moment. If the moment lies in the ribbon plane, the parameter $A_{23}$ equals four and if it is perpendicular to the ribbon plane, it becomes zero. For nanocrystalline sample of NANOPERM, the $A_{23}$ parameter shows a slightly decreasing tendency with exposure time (Fig. 2). Both described features (change in hyperfine field as well as in orientation of net magnetic moment) reflect changes in the shortrange order of amorphous component resulting from a growth of the crystalline component and corrosion products.

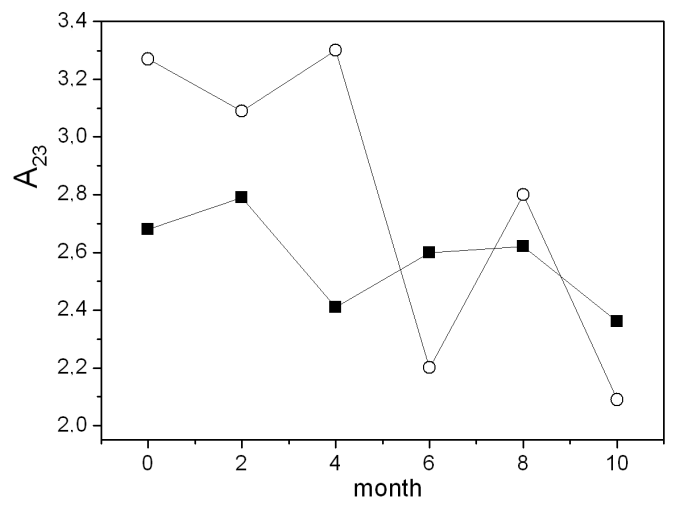

Fig. 2. Mössbauer parameter $A_{23}$ as a function of exposure time. Squares - NANOPERM; circles - FINEMET.

Similar tendency of the $A_{23}$ parameter was observed for the FINEMET-alloy as shown in Fig. 2. Changes of all other Mössbauer parameters for FINEMET were not significant (within the experimental error), which indicates very good corrosion resistance. This can be attributed mainly to the composition of the alloy leading to creation of a protective passive film due to the presence of silicon in the sample.

Further investigation was focused to the NANOPERM-type alloy with nominal composition $\mathrm{Fe}_{76} \mathrm{Mo}_{8} \mathrm{Cu}_{1} \mathrm{~B}_{15}$ because the presence of molybdenum could influence corrosion properties. In atmospheric environment, the changes in the spectra occurred very early. The $\gamma$-FeOOH compound participated on the spectrum composition with almost $9 \%$ after one month and $23 \%$ after two months. Concerning the magnetic microstructure, the decrease in the average hyperfine field was observed from $14.18 \mathrm{~T}$ to $12.91 \mathrm{~T}$. The $A_{23}$ parameter decreased from 1.53 to 1.10 , which indicates the net magnetic moment rotation out of the ribbon plane. The Mössbauer spectra of untreated and treated (2 months) sample are shown in Fig. 3.

Ambient conditions are expected to induce similar qualitative degradation, however with lower rate, which is less suitable for experimental observation.

Similar behaviour was observed for radiation corrosion after neutron irradiation. The most sensitive parameter was direction of the net magnetic moment and change of the average field and magnetic field distribution. After high-fluency 


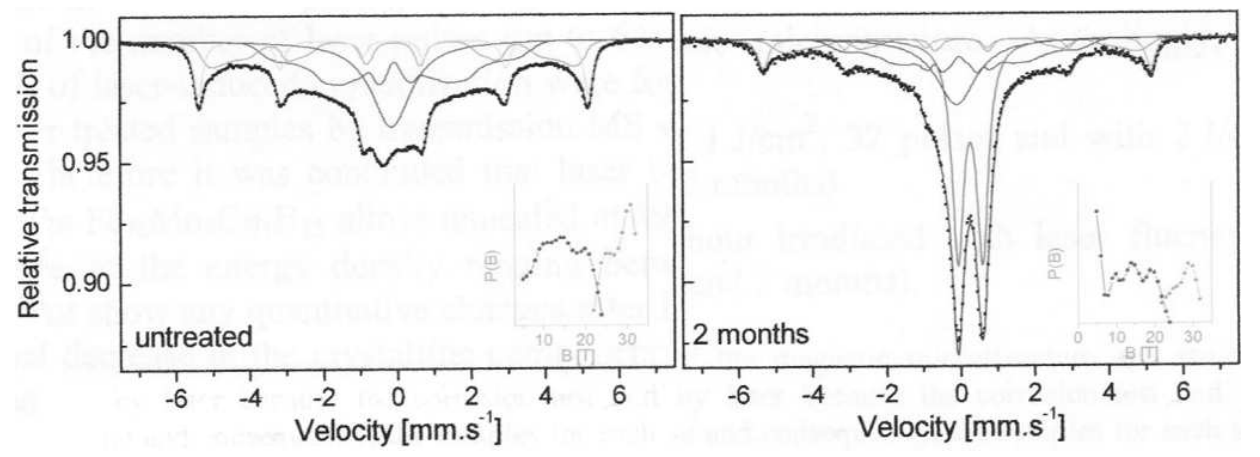

Fig. 3. Mössbauer spectra of $\mathrm{Fe}_{76} \mathrm{Mo}_{8} \mathrm{Cu}_{1} \mathrm{~B}_{15}$ including the $P(B)$ distributions.

irradiation $\left(10^{19}\right.$ neutrons $\left./ \mathrm{cm}^{2}\right)$, new phases were created and the magnetic parameters changed substantionally [4].

\section{Conclusions}

Concerning magnetic microstructure, mostly occurring tendency was the rotation of the net magnetic moment out of the ribbon plane. This behaviour might result from the internal stresses produced during corrosion treatment. In addition, the observed changes in the average hyperfine field and its distribution having different strength for each individual case indicated the changes of the microscopic magnetic properties caused by corrosion damage. Similar response is expected for corrosion caused by ion-beam irradiation that is in progress.

\section{Acknowledgments}

The authors thank to P. Svec for supplying the samples. The work was supported by grants VEGA 1/3189/06, EU/INTAS/07 and INTAS 06-10000128683.

\section{References}

[1] Y. Yoshizava, Scr. Mater. 44, 1321 (2001).

[2] C.A.C. Souza, J.E. May, I.A. Carlos, M.F. de Olivera, S.E. Kuri, C.S. Kiminami, J. Non-Cryst. Solids 304, 210 (2002).

[3] N.A. Mariano, C.A.C. Souza, J.E. May, S.E. Kuri, Mater. Sci. Eng. A 354, 5 (2003).

[4] J. Sitek, J. Degmova, in: Proc. NATO Advanced Research Workshop MSMS, Senec, Eds. M. Miglierini, D. Petridis, Kluwer Academic Publishers, Netherlands 1999, p. 273.

[5] K. Sedlackova, Ph.D. Thesis, STU Bratislava 2005. 